\title{
An immune memory and negative selection to visualizing clinical pathways from electronic health record data
}

\author{
Mouna Berquedich', Oulaid Kamach ${ }^{2}$, Malek Masmoudi ${ }^{3}$, Laurent Deshayes ${ }^{4}$ \\ ${ }^{1,2}$ Abdelmaleksaâdi University, Laboratory of Innovative Technologies (LTI), Morocco \\ ${ }^{3}$ Jean Monnet University, Laboratory of industrial engineering, France \\ ${ }^{4}$ University polytechnique de Benguérir, Morocco
}

\begin{tabular}{l} 
Article Info \\
\hline Article history: \\
Received Jul 1, 2019 \\
Revised Dec 9, 2019 \\
Accepted Jan 15, 2020 \\
\hline
\end{tabular}

Keywords:

EHR

Hospital environment

AIS

Negative selection

Immune memory

\begin{abstract}
Clinical pathways indicate the applicable treatment order of interventions. In this paper we propose a data-driven methodology to extract common clinical pathways from patient-centric Electronic Health Record data (EHR). The analysis of patient's, can lead to better regarding pathologies. The proposed algorithmic methodology consist to designing a system of control and analysis of patient records based on an analogy between the elements of the new EHRs and the biological immune systems. The detection of patient profiles ensured by biclustering Matrix. We rely on biological immunity to develop a set of models for structuring knowledge extracted from EHR and to make pathway analysis decisions. A specific analysis of the functional data leds to the detection of several types of patients who share the same EHR information. This methodology demonstrates its ability to simultaneously processing data, and is able to providing information for understanding and identifying the path of patients as well as predicting the path of future patients.
\end{abstract}

Corresponding Author:

Mouna Berquedich,

Abdelmaleksaâdi University,

Laboratory of Innovative Technologies (LTI), Tangier, Morocco.

Email: berquedich.mouna@gmail.com

\section{INTRODUCTION}

The use of EHRs that has developed around the world, more and more hospitals and health care providers are recognizing its benefits. However, it is difficult to identify the specific factors contributing to improved care. One of the main problems in this area is whether the information provided by the EHR is effective (in terms of better care and time) and whether it helps doctors in their decision-making. The results of several work on EHRs suggest that the use of EHRs improves medical decision-making in terms of accuracy of diagnosis and admission of correct decisions, as well as better quality of care. This study contributes to medical decision-making in that the results show how access to EHR can improve patient care and save time and money. the objective of this work is to rely on the EHR to increase the cooperation and willingness of medical personnel to adopt a computer system by demonstrating its contribution to the correction of medical diagnostics. The system developed is based on a set immune concepts and mechanisms, such as the negative selection algorithm used to monitor, and an immune memory algorithm used to select the appropriate pathway detection strategy to respond to detected disturbances. The proposed system maintains the performance of a clinical decision support system at a high level. The combination of negative selection mechanisms and immune memory gives the system the ability to recognize disturbances and select appropriate decisions. In order to improve the system described in this article in, other immune concepts may be used. These concepts 
inspired by the biological immune system, such as the theory of danger, can be studied to take into account the influence of a bad diagnosis on the prediction of the paths of other patients.

The patient pathway could also refer to the succession of steps in the handling of a patient within a hospital [1]. This may refer to a sequence of procedures (e.g. clinical examination, laboratory dosage, biopsy, and then surgery), a sequence of clinical stages (e.g. inflammatory, proliferative, and maturation phases), or a sequence of medical units (e.g., emergency unit, surgery, intensive care, conventional hospitalization, rehabilitation care, and then housing unit) [1]. In this context, the unprecedented availability of hospitals data gives the opportunity to improve decision-making and to discover best practices for healthcare delivery [2]. This article presents a case study of EHRs-the study of the management process of the hospital medical EHR and the construction of futur Pathaways of patients in order to identify patient's profiles from the EHR data of the already occurred patients pathways.

\subsection{EHR: Benefits and Practice}

Electronic Health Records (EHR) is a digital collection of patient health information. EHR is increasingly being implemented in many developing countries. These records can be shared through well connected network across different health care settings. EHR includes demographic and personal statistics like age, weight, and billing information as well as vital signs, family history, medication and allergies history, immunization status, laboratory test results, and radiology images. EHR systems are designed in such a way to reduce paper medical records by storing data accurately and legible in a digital format. However digitally health records reduce the risk of data replication as file can be shared across the different health care systems and can be easy updated which reduce the risk of lost paperwork. EHR programs directly benefit the physicians, patients, and obviously the hospital management authority. EHR system used for population based studies and effective when extracting medical to predict possible trends in healthcare system.

\subsection{Benefits with HER}

EHR is a great tool to manage lengthy and labor-intensive paperwork more efficiently and thereby significantly reduce the cost of transcription, re-filling, and storage. EHR enables patient management with enhanced and accurate reimbursement coding. Since the software has all patient-related information it significantly reduces the occurrence of a medical error and also helps in the improvement of patient health with better management of the diseases. Here we discuss five important benefits of EMR vs. Paper Medical Records [4] . 1. Costs: To start the EHR the initial costs is higher due to large and digitally setting of IT network but the costs over time will decrease significantly. While manually storage of paper records require more personnel to manage and maintain paper files, accesses and organize countless documents which increase cost substantially over the time. EHR can save man power, time and physical storage space which reduced the cost in long run. 2. Storage: Electronic health records can be stored in a secure cloud, providing easier access by those who need them, however paper medical records required large warehouses for storage. Paper records are not only taken up space, but they are not environmentally friendly and tend to decay, when handled by many individuals over time, which increased the cost of storage. 3. Security: Security is a great concern for both paper and electronic storage system; both are equally susceptible to security threats. If a facility stores records electronically without proper and effective security systems they are vulnerable to access by unauthorized individuals which can be misuse the information. If records are stored in paper form, they can be lost or damage or stolen due to human error. Natural disaster such as a fire or flood also plays an important role in the concern the security of health records. 4. Access: Accessibility of electronic health records take clear advantage over paper health records. Digital health records allows healthcare professionals to access the information instantly, whenever and wherever they need almost, which makes healthcare professionals more efficient, however paper medical records to be shared with healthcare professional required physically provided to them or scanned and sent via email, it is a time-consuming which increase the cost. 5. Readability and Accuracy: Electronic health records are often written with the use of standardized abbreviations which make them more accurate and readable across the globe which decreased the chance for confusion however handwritten paper medical records may be poorly legible, which can contribute to medical errors [5]. Paper medical records provide insufficient space for healthcare professionals to write all necessary information.

\subsection{Better practice management with EHR}

In terms of appointment management, EHR performs the excellent task. EHR improved medical practice management through integrated scheduling systems that link appointments directly to progress notes, automate coding, and manage claims. This platform smoothly handles queries about the patient condition and manages graphs, specific to each and every patient. EHR enhanced communication with other multidisciplinary physicians, laboratories, and between different hospitals which enables faster patient service. Since it"s a digital platform with online connectivity doctors can access the patient information anytime anywhere 
and assigned task to support providers which includes labs, and other physicians [6]. Follow checkups are often an integral part of better patient care; with EHR, automated checkups are easily scheduled by the electronic program. Since the program is integrated with test reports and images, timely access is readily enabled. Moreover, such test can be ordered through this multipurpose EHR system. Moreover, such system prevents unnecessary duplication of medical testing. The best part of such electronic records is that they are integrated with national and international disease database and registries. Thus, it helps the physicians track the epidemiological status of the current disease under treatment and be prepared for emergencies [7].

\section{LITERATURE REVIEW}

\subsection{Artificial immune systems}

The Artificial Immune System field has been inspired from natural immune system of several species. Ambitiously, to develop systems that operate in environments similar to constraints faced by the natural immune system. De Castro and Timmis defines the AIS as "the adaptive systems, inspired by the theories of the immunology, as well as the functions, the principles and the immune models, in order to be applied to the resolution of problems" [8].

The immunity is subdivided into two distinct systems: innate immune system and adaptive immune system. The adaptive immune system has three principal processes [9]: negative selection, clonal selection and immune network. Whereas, Natural Dendritic Cells are the link between the innate and adaptive immune system.

\subsection{Clonal selection}

There are many algorithms based on clonal selection in the literature, most of which have been applied to optimization problems (e.g., CLONALG [10] and opt-IA is used in [11], and the algorithm to B cells in [12]), and multi-objective optimization (see [13]). From a computational point of view, the idea of clonal selection leads to algorithms that iteratively improve the possible solutions to a given problem through is almost the same as clonal selection, except that there exists a mechanism of deletion that destroys the cells having a certain inception of affinity amidst.

\subsection{Algorithm of negative selection}

The basic idea of a negative selection algorithm is to generate a number of detectors in the complementary set $\mathrm{N}$, then applying these detectors to classify the new data as auto or non-auto [17]. Negative selection algorithms have been very widely used in aquatic invasive species research and have undergone many improvements over the years [18]. Clonal selection algorithms are mostly used as optimizing algorithms. They use fewer classifications. Thus, clonal selection principles first appeared in 1959 [19]. Artificial immune systems algorithms used for classification are considered as classifiers, since they combine the output of many simple classifiers all together.

\section{AIS METHODS}

The Two types of cells involved to recognize presenting pathogens are; T-cells and B-cells. The population of these present cells in the bloodstream is responsible for recognizing and destructing the pathogens. The population acts collectively. It is capable to identify new pathogens through two training methods: negative selection and clonal selection. Through the process of negative selection, the NIS is able to protect the host organism tissues from being attacked by its own immune system. Some cells generate detectors that recognize proteins, which are present on the surfaces of cells. The detectors are called "antibodies". They are randomly created. Before the cells become fully mature, they are "tested" in the thymus. The thymus, an organ located behind the sternum, is able to destroy any immature cells that identify the tissues of the organism as "non-self" [20]. The process of negative selection maps therefore the negative space of a given class such as given examples of the "self" class. The negative selection algorithm first appeared in 1994 [21]. Using the clonal selection, the NIS is apt to adjust itself to provide the most efficient response against pathogen attacks. Clonal selection happens when a cell detector finds already seen pathogen in the organism, it clones itself then to start the immune response. The cloning process, however, introduces small variations in the pattern that the cell detector recognizes. The number of the clones created by a cell detector is proportional to the new pathogen cell "affinity". It is a measured manner to detect to which extend the cell matches the pathogen. The amount of variation allowed in the clones is negatively proportional to the affinity; the cells with the most affinities are mutated less. The clonal selection algorithms are similar to the natural selection systems. And the clonal selection algorithm is therefore similar to the genetic algorithms based on the natural selection [22, 23]. 
Nevertheless, clonal selection algorithms have less parameters than genetic algorithms, and potentially, they do not require complex functioning operations. Clonal selection algorithms are mostly used as optimizing algorithms. There have been a few of them used for classification. The clonal selection principle first appeared in 1959 [24, 25]. Artificial immune systems algorithms applied for classifications are considered to being classifiers; they combine the output of many simple classifiers.

\subsection{System overview}

The main objective of our work is to develop a vital supporting tool for hospital decision-makers to strengthen the quality of their decisions face of the massive flow of patients. The fundamental idea is to detect traces in the database, and to help executives by identifying bad scenarios utilizing AIS techniques, especially negative and clonal selection. The analogy between the principle of the natural immune system and the problem as proposed (Table 1) has prompted us to develop our system.

Table 1. Analogy Between the Natural Immune System Principle and the Developed System of EHR

\begin{tabular}{ll}
\multicolumn{1}{c}{ Natural Immune System } & \multicolumn{1}{c}{$\begin{array}{c}\text { Artificial Immune System applied in our } \\
\text { Hospital emergency context }\end{array}$} \\
\hline Body & EHR \\
Self & Normal Pathway of patient \\
Infected Cell & Disturbed Pathway \\
Non-self (antigen) & False Pathway \\
antibody & Control Decisions \\
Lymphocyte (B) & Combination of control decision for detected \\
& disturbance \\
Affinity & Adequacy between the correction actions and \\
& disturbance. \\
Memory Cells & Data base \\
Response Strategy & Immune Memory Based Algorithm \\
\hline
\end{tabular}

\subsection{Self-cell representation}

Self-cells represent normal situations of EHR. In this paper, we suggest a model to represent and structure knowledge related to normal situations of EHR. The model includes five attributes as presented in equation (1):

$$
\begin{aligned}
& \mathrm{SE}=\left\{\mathrm{D}, \operatorname{Pr}_{\mathrm{j}}, \mathrm{P}_{\mathrm{j}}, \mathrm{M}_{\mathrm{j}}, \mathrm{D}_{\mathrm{j}}\right\} \\
& \mathrm{D}_{\mathrm{j}}: \text { Date } \\
& \operatorname{Pr}_{\mathrm{j}}: \text { Purpose } \\
& \mathrm{P}_{\mathrm{j}}: \text { Procedure } \\
& \mathrm{M}_{\mathrm{j}}: \text { Medication } \\
& \mathrm{Dg}_{\mathrm{j}}: \text { Diagnosis }
\end{aligned}
$$

\subsection{Antigen represntation}

A disturbance is any kind of event that affects a pathway of patient. We characterize a disturbance as a vector of patient $i$ with 5 attribute describing the affected pathway:

\section{Vi \{ Date Purpose Procedure Medication Diagnosis \}}

$\mathrm{e}_{\mathrm{lk}},(\mathrm{k}=1, \ldots, \mathrm{kl}, \mathrm{l}=1, \ldots, 1)$, represents a set of $\mathrm{kl}$ events of 1 specific types, referred to as 'scenario' hereafter, that occurs during a patient's medical visit. In Table 1, the "Office" event corresponds to the type of contact, the "CKD Stage 3" event to the type of diagnosis, the "Diuretics" event to the type of medication, and the "Renal" type of ultrasound; procedure [26]. For example, in Table 1, the record of patient 1 can be transformed using the scenario shown below in Table 2. The procedure, treatment and diagnostic scenarios are named respectively $\mathrm{P}_{\mathrm{j}}, \mathrm{M}_{\mathrm{j}}$ and $\mathrm{D}_{\mathrm{j}}, \mathrm{j} \in \mathrm{Z}+$.

Our goal is to identify common sequences from data that can constitute clinical pathways. To do this effectively, we introduce an element called immune memory and negative selection to represent unique visitor content. For each patient we will have a unique combination: Purpose of visit, procedure, medication and diagnosis. Negative selection to reduce multidimensional visit records so that they can be represented as a 
sequence of visits ordered by date of visit. Each patient has one and only one sequence, starting with the first visit recorded in the EHR and ending with the last visit.

Table 2. Description of Visit (A)

\begin{tabular}{llllll}
\hline Patient & Date & Purpose & Medication & Procedure & Diagnosis \\
\hline 1 & $\mathrm{xx} / \mathrm{yy} / 2012$ & $\mathrm{P} 1$ & $\mathrm{M} 1$ & $\mathrm{~N} / \mathrm{A}$ & $\mathrm{D} 1$ \\
1 & $\mathrm{xx} / \mathrm{yy} / 2013$ & $\mathrm{P} 2$ & $\mathrm{M} 2$ & $\mathrm{P} 1$ & $\mathrm{D} 2$ \\
1 & $\mathrm{xx} / \mathrm{yy} / 2015$ & $\mathrm{P} 3$ & $\mathrm{M} 3$ & $\mathrm{~N} / \mathrm{A}$ & D1 \\
1 & $\mathrm{xx} / \mathrm{yy} / 2018$ & $\mathrm{P} 4$ & $\mathrm{M} 4$ & N/A & D3 \\
\hline
\end{tabular}

Table 3. Description of Visit (B)

\begin{tabular}{llllll}
\hline Patient & Visit Date & Visit Purpose & Procedure & Medication & Diagnosis \\
\hline 1 & $24 / 09 / 2012$ & P1 & N/A & ACE inibitors & CKD stage 4, hypertension \\
1 & $5 / 2 / 2013$ & P2 & Renal ultrasound & ACE inhibitors dueritic & AKI,CKD Stage 4hypert \\
1 & $3 / 1 / 2015$ & P3 & N/A & ACE inhibitors, dueritic statin & CKDS \\
1 & $3 / 6 / 2018$ & P4 & N/A & ACE Inhibitors Dureritic statin & $\begin{array}{l}\text { AKI,CKD } \\
\text { Stage 5, hypertension }\end{array}$ \\
\hline
\end{tabular}

The developed Immune Memory based Algorithm (IMA) works according to the following steps:

a. Representation of B cells

The control strategies are represented by the "B cells" that allow the recognition process and neutralize the antigen detected in the sphere concerned. They aggregate with one or more decision controls (antibodies) that react each time the disturbance occurs. Therefore, the system must create a B cell identical to each detected antigen. Equation (1) above, illustrates the definition of independent B-cells that receptors are a prerequisite that has the structure similar to that of the antigen that has been described bellow (look at the Equation 3). The set of receptors, epitopes and antibodies is equal to the B cells. According to the model suggested in, a control decision can have the value 0 or 1 . Whether the antibody is operated or activated, the similar epitope indicates the value 1 and the value 0 is assigned to it otherwise. Epitopes are the activators of antibodies in these cases.

\section{b. Step 1: Learning}

This step aims to produce sets of non-self cells using periodic comparisons between normal situations (self cells) and the state of the new EHR. We highlight matching ratios to quantify existing distances in given situations, using data collected from the facts. These measures are designated by SE and the elements of the set $\mathrm{S}$ with respect to testing their similarities. The matching rate is calculated mathematically by adapting equation (3).

$$
\operatorname{Mat}(S i, S E)=\frac{100}{5} \times \sum_{1}^{5} \alpha i
$$

The «Mat ( $\mathrm{Si}, \mathrm{SE}) »$ represents the corresponding percentage $(\%)$. The "Si" indicates a normal situation among the set "S". SE determines a situation. Attribute values are extracted from the database. $\alpha \mathrm{i}$ is calculated using the following method (4):

$$
\alpha i=\left\{\begin{array}{c}
1 \text { if } S^{j}=S_{i}^{j} \\
0 \text { else }
\end{array}\right.
$$

The "SEj" is the "j" th attribute of an "SE" situation captured from the database (look (1)). The The «Sij» is the "j" th attribute of an "SE" situation captured from the database (look (1)). The «Sij» is the "j" th attribute of the "i" th situation extracted from the set $\mathrm{S}$. When the adequacy rate is lower than the coverage already fixed " 1 " e; therefore, the situation is classified as abnormal and will then be added to the R set of patterns. To close this process, the set $\mathrm{R}$, this includes various types of deviant pathway, displays the most significant abnormal pathway. In fact, it is used in the next steps to identify the disturbances that have occurred. Figure 1 draws the components of the set $\mathrm{R}$. 


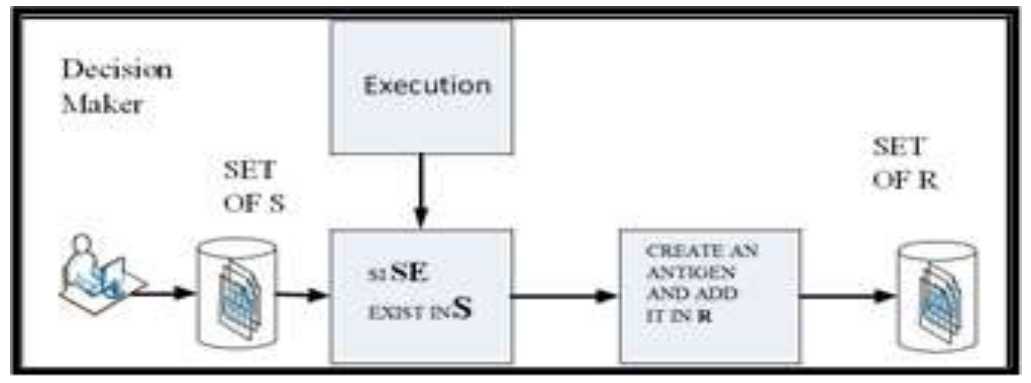

Figure 1. The Suggested Negative Selection Algorithm (NSA)

\section{c. Step 1: Monitoring}

The given $\mathrm{R}$ which represents all the patterns is constructed in the step indicated above. The PS is designed to progressively read the values from the databases and measure the similarities with the situation of abnormalities of the R-set, non-auto-cellular cells. Equation (4) can be used as a determinant of the matching rate which groups all the abnormal situations of the set $\mathrm{R}$ with a current situation. Whenever the actual situation is the same as that already coded in the set R, that is, the matching rate is much higher than the fixed interval, it follows that perturbation is identified and a specific antigen created. The reaction and response will then be activated by the PS.

\section{RESULTS AND ANALYSIS}

This section presents the implementation of the EHR management system and discusses the results obtained. The proposed decision support system is implemented with the JAVA programming language. To evaluate the system, a hospital database with a history of four years with more than 100,000 EHR patients. We have simulated the different cases presented below. To construct the $\mathrm{S}$ set, we used a real patient records database (EHR).

Table 4. Illustrations of an example sample EHR on our system

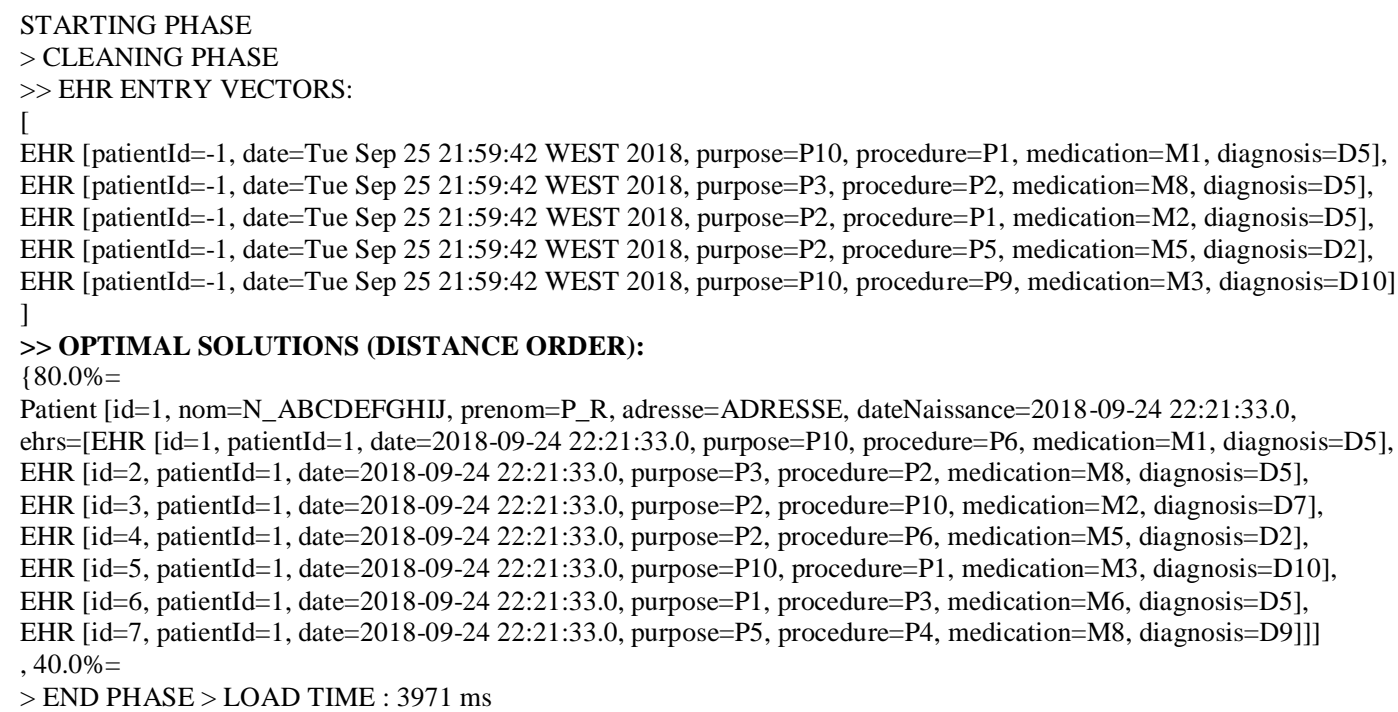

The results above show the phase of the regrouping of the relevant information in the form of a vector of five components, thereafter an analysis is conducted according to two immune processes to communicate to the hospital decision maker the path closest to the path of the patient entered. . The execution phase included a grouping and vector standardization compatible with the input patient information. Subsequently two immune algorithms as previously explained the negative selection and immune memory for the display of the optimal solution that responds to the patient input. 
The goal has been achieved, by offering the hospital decision maker a tool for detecting the patient's journey, in broad and unstructured information. The most important is the fact of constantly feeding the database R, containing the erroneous solutions in order to avoid the medical errors of the old patients during their medical journey to the recent patients who share the same pathology and symptoms with them.

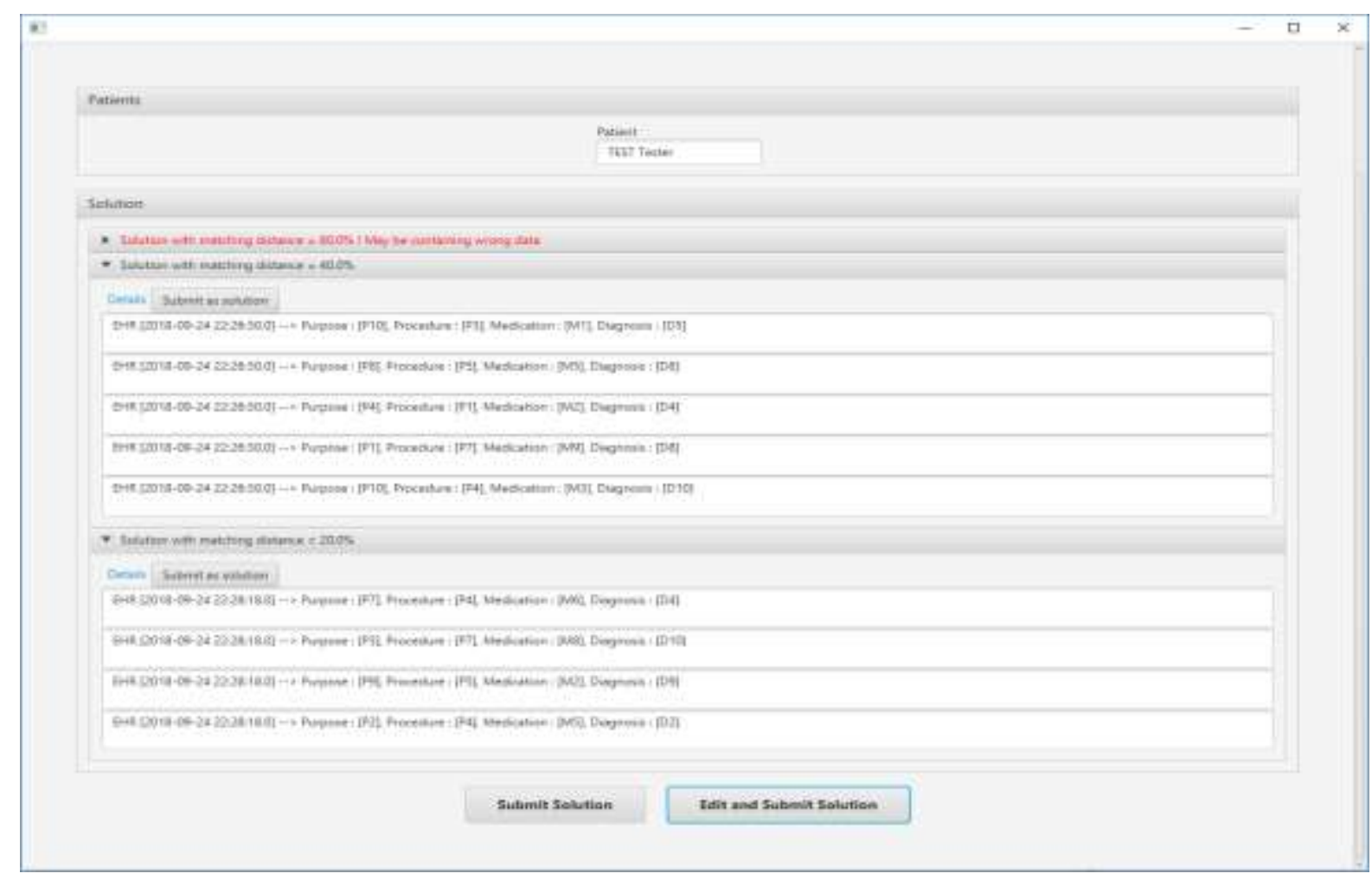

Figure 2. Results of EHR Selection by Affinity Performance

As illustrated in the previous figures, we realized the efficient and effective decision support system, allowing the filtering and the analysis of the data of the EHRs of the different patients while adopting the immune principles of the memory cells and of negative selection.

\section{CONCLUSION}

We are designing a patient record analysis system, based on an analogy between the elements of the new HRTs and the biological immune systems. Patient profiles are detected by the memory cells. We rely on biological immunity to design a set of models that can be used to structure knowledge about EHRs and pathway analysis decisions. A specific analysis of functional data, led to the detection of several types of patients, who share the same information on their EHR. This methodology demonstrates its ability to simultaneously process data. It is able to provide information for the understanding and identification of patients' pathways as well as for predicting the path of future patients.

\section{REFERENCES}

[1] J. E. Barr, J. Cuzzell. "Wound care clinical pathway: a conceptual model". Ostomy Wound Manage, 42(7), pp.1824, 1996.

[2] B. T. Denton. "Handbook of healthcare operations management". New York: Springer, 2013.

[3] A. Kellermann, "Crisis in the Emergency Department," New England Journal of Medicine, Vol.355, No.13, pp.13001303, 2006.

[4] F. Harrou, F. Kadri, S. Chaabane, C. Tahon, and Y. Sun, "Improved principal component analysis for anomaly detection: Application to an emergency department," Computers \& Industrial Engineering, Vol.88, pp.63-77, 2015. 
[5] D.G. Goldberg, A.J. Kuzel, L.B. Feng, J.P. DeShazo, L.E. Love, EHRs in primary care practices: benefits, challenges and successful strategies, Am. J. Manage. Care. 18 (2012) e48-e54.

[6] A. Takian, A. Sheikh, N. Barber, We are bitter, but we are better off: case study of the implementation of an electronic health record system into a mental health hospital in England, BMC Health Serv. Res. 12 (2012) 484.

[7] B. Jarvis, T. Johnson, P. Butler, K. O'Shaughnessy, F. Fullam, L. Tran, et al., Assessing the impact of electronic health records as an enabler of hospital quality and patient satisfaction, Acad. Med.: J. Assoc. Am. Med. Colleges 88 (2013) 1471-1477.

[8] F. Kadri, C. Pach, S. Chaabane, T. Berger, D. Trentesaux, C. Tahon, and Y. Sallez, "Modelling and management of strain situations in hospital systems using an orca approach," in Proceedings of 2013 International Conference on Industrial Engineering and Systems Management (IESM). IEEE, pp. 1-9, 2013.

[9] F. Kadri, F. Harrou, S. Chaabane, and C. Tahon, "Time series modelling and forecasting of emergency department overcrowding," Journal of medical systems, vol. 38, no. 9, pp. 1-20, 2014.

[10] F. Harrou. Y. Sun. F. Kadri. S. Chaabane, and C. Tahon. "Earlv detection of abnormal patient arrivals at hospital Emergencv department." In 2015 International Conference on Industrial Engineering and Systems Management (IESM), pp. 221-227. IEEE, 2015.

[11] F. Kadri, S. Chaabane, and C. Tahon, "A simulation-based decision support system to prevent and predict strain situations in emergency department systems," Simulation Modelling Practice and Theory, vol. 42, pp. 32-52, 2014.

[12] P. Carey, G. Cuthbert, R. Dang, B. Greystoke, A.McGregor, R. Oakes, and J. Wallis, "The North of England haemato-oncology diagnostic service (NEHODS): A more devolved and inclusive approach to integrated reporting facilited by an IT system (Haemosys) networked to local information management systems (LIMS) in all participating regional hospitals", British Journal Of Haematology, Vol.173, No.1, 2016.

[13] J. V.de Carvalho, Á.Rocha, and J.Vasconcelos, "Towards an Encompassing Maturity Model for the Management of Hospital Information Systems", Journal of Medical Systems, Vol.39, No.9, pp.99, 2015.

[14] C. Virenque. Large influx of injured people in hospital(pp. 712-715). Hôpital Purpan, TSA 40031, 31059 Toulouse cedex 09, France Elsevier, 2016.

[15] L. N. De Castro, and J. Timmis, "Artificial immune systems: a novel paradigm to pattern recognition", Artificial Neural Networks in Pattern Recognition, Vol.1, pp.67-84, 2002.

[16] J. Timmis, A. Hone, T. Stibor, and E. Clark,"Theoretical Advances in Artificial Immune Systems",Theoretical Computer Science, Vol.403, No.1, pp.11-32, 2008.

[17] U. Aickelin, and D. DasGupta, “Artificial immune systems”, Search Methodologies, pp.375-399, 2005.

[18] L. N. De Castro, andF. J. Von Zuben, "The Clonal Selection Algorithm With Engineering Applications", Paper presented at The Workshop on Artificial Immune Systems and Their Applications, Las Vegas, USA, 2000.

[19] L. N. De Castro, and F. J. Von Zuben,"Learning and Optimization Using the Clonal Selection Principle", IEEE Transactions on Evolutionary Computation, Vol.6, No.3, pp.239-251, 2002.

[20] N. K. Jerne, “Towards a Network Theory of the Immune System”, Ann Immunol, Vol.125, pp.373-389, 1974.

[21] J. Greensmith, U. Aickelin, and G. Tedesco, "Information Fusion for Anomaly Detection with the Dendritic Cell Algorithm". Information Fusion Journal, 11(1), pp.21-34, 2010.

[22] J. Greensmith, U. Aickelin, and S. Cayzer, "Introducing Dendritic Cells as a Novel Immune-Inspired Algorithm for Anomaly Detection”,International Conference on Artificial Immune Systems, Springer, Berlin, Heidelberg, pp.153167,2005

[23] M. Burnet, "The Clonal Selection Theory of Acquired Immunity", Nashville, Vanderbilt University Press, 1959. doi:10.5962/bhl.title.8281.

[24] B. Schmidt, A. Al-Fuqaha, A. Gupta, and D. Kountanis, "Optimizing an artificial immune system algorithm in support of flow-Based internet traffic classification", Applied Soft Computing, Vol.54, pp.1-22, 2017.

[25] S. Forrest, A. Perelson, L. Allen, R. Cherukuri, "Self-nonself discrimination in a computer",In: Research in Security and Privacy, 1994. Proc. 1994 IEEE Computer Society Symposium on, pp.202-212, 1994.

[26] S. Mnif. S. Elkosantini. S. Darmoul. and L. Ben Said. "An immune multi-agent based decision support svstem for the control of public transportation svstems." In International Conference on Practical Applications of Agents and Multi-Agent Systems, pp. 187-198. Springer, Cham, 2016. 